\title{
Painful leg and missing pulses: a case report
}

\author{
C W M Chan, J I Wilson, A Myatt, P N Roberts
}

\begin{abstract}
Popliteal artery entrapment syndrome (PAES) is a rare but potentially limb threatening anatomical anomaly occurring predominantly in young adults. We report a case of a 14 year old boy who presented with limb threatening ischaemia requiring urgent surgery. The pathology, presentation, and management are discussed.

(Arch Dis Child 2000;83:362-363)
\end{abstract}

Keywords: popliteal artery entrapment syndrome; acute limb ischaemia

Children with lower limb pain pose a difficult diagnostic challenge and not infrequently present to the emergency department. The differential diagnosis is quite broad but fortunately few conditions require urgent treatment. ${ }^{1}$

Entrapment of the popliteal artery is a recognised cause of ischaemic lower limb pain in young adults aged $30-40$ years and is caused by either congenital abnormalities of muscle insertion or acquired hypertrophy of surrounding structures.

We report a case of a 14 year old boy who presented with acute lower limb pain caused by popliteal artery entrapment syndrome (PAES). We highlight this as an important but rare cause of lower limb pain in children that needs to be recognised and urgently treated if loss of limb is to be averted.

\section{Case report}

A previously well 14 year old Anglo-Asian boy presented with a two day history of right foot pain, worse at night, and associated with pallor and pain of the great toe which was relieved by hanging his foot over the edge of the bed. $\mathrm{He}$ did not describe a previous history of intermittent claudication, nor have any known cardiac or haematological disorders.

On examination, he had a cold, pale, pulseless right foot with absent popliteal and ankle pulses. He was apyrexial, in sinus rhythm, and had a normal cardiac examination. The contralateral limb was unremarkable, as were the other systems. He had a normal haemoglobin, and white cell and platelet count. A full coagulation, vasculitic, and prothrombotic screen did not reveal any abnormalities. Clinically, he had occluded his popliteal artery. $\mathrm{He}$ was heparinised and an urgent femoral angiogram showed an occlusion of his right popliteal artery (fig 1). At this stage his foot deteriorated and an urgent exploration was performed.

At operation, the popliteal artery was found within the medial tendinous head of the gastrocnemius muscle with resultant occlusion of the vessel at this level. Thrombus was present down to the popliteal trifurcation. This was corrected with a reversed long saphenous vein bypass graft from the distal superficial femoral artery to the infrageniculate popliteal artery and the distal vessels embolectomised.

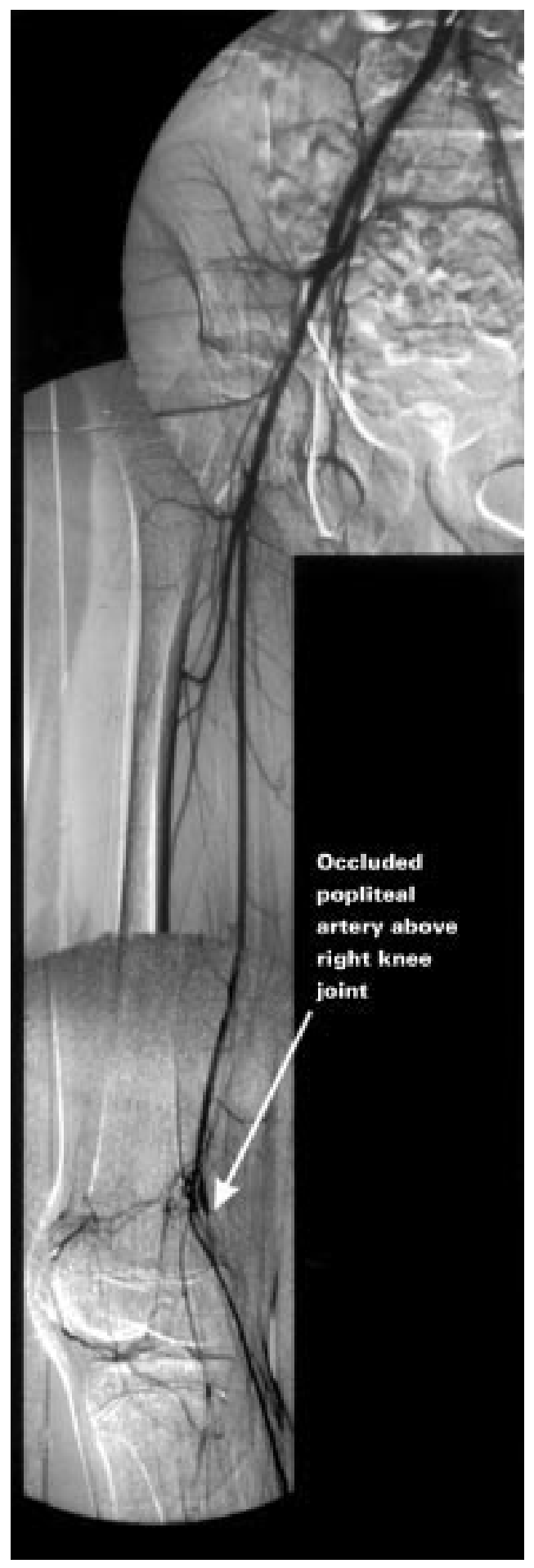

Figure 1 Right femoral angiogram of the patient. 
He had an uneventful postoperative recovery, and at follow up two months later his right leg was functionally back to normal without any claudication. An arterial duplex scan of the left leg did not reveal any abnormality.

\section{Discussion}

Popliteal artery entrapment was first recognised in 1879 as a developmental abnormality of the popliteal artery and gastrocnemius muscle. ${ }^{2}$ The popliteal artery normally runs with the vein down the centre of the popliteal fossa between the two heads of the gastrocnemius muscle. In the entrapment syndrome, the popliteal artery has an aberrant course and is compressed by the medial head of the gastrocnemius muscle in a variety of ways. ${ }^{3}$ This causes compression and with repeated pulsation damages the artery over a period of time. Autopsy series suggest that the incidence of anatomical abnormality of the popliteal artery may be as high as $3.5 \%,{ }^{3}$ although the incidence of clinically significant entrapment is thought to be $0.17 \%,{ }^{4}$ with a male:female ratio of $9: 1$.

Most patients present in early adulthood with intermittent claudication, or complain of aching legs after exercise. PAES presenting as intermittent claudication has been described in a patient as young as 15 years old. ${ }^{5}$ However, we believe that this is the first reported case of a paediatric patient presenting with an acutely ischaemic limb caused by popliteal artery thrombosis from the entrapment syndrome.

Ischaemic symptoms in children and young adults can often be misinterpreted as being musculoskeletal in origin. Even in specialist paediatric texts acute limb ischaemia may not be considered among the numerous causes of a limping child. ${ }^{1}$ Possible aetiology includes embolic syndromes (for example, infective endocarditis), thrombophilia and hypercoagulable states (for example, disseminated intravascular coagulation), and small vessel disease (for example, vasculitis). In an otherwise well patient, clinical suspicion should be directed at popliteal artery entrapment syndrome. Angiography is useful in providing a "road map" of the aberrant anatomy although non-invasive duplex scanning is increasingly being employed as it shows both morphology as well as blood flow dynamics.

Treatment is surgical. Complete division of the abnormal muscle and transposition of the popliteal artery laterally may be all that is required in an undamaged artery. If the entrapped artery is damaged or occluded, then a bypass procedure using autologous vein is the procedure of choice. Catheter thromboembolectomy with intra-arterial thrombolysis has been reported as a viable option before surgical transposition if the situation permits. ${ }^{6}$ Surveillance of the contralateral popliteal artery should be carried out as a proportion of patients have bilateral entrapment, which, if present, should always lead to surgical correction of the asymptomatic side. This can be easily performed by means of duplex scanning with passive dorsiflexion and plantar flexion to induce vessel compression.

The combination of early age and first presentation with a critically ischaemic limb in our patient does raise concern, especially in an emergency setting when non-vascular surgeons are the first point of contact. In considering the differential diagnosis of a child who limps, refuses to walk, or complains of limb pain, acute vascular conditions are rarely suspected by the emergency physician. While acute limb ischaemia in a child is rare, the threat of devastating limb loss should make this an important differential diagnosis. The absence of the dorsalis pedis and posterior tibial pulses should alert one to this possibility and an urgent referral to a vascular surgeon should be made.

1 Lawrence LL. The limping child. Emerg Med Clin North Am 1998;16:911-28.

2 Stuart TPA. Note on a variation in the course of the

3 Gopliteal artery. F Anat Physiol 1879;13:162. Gibson MH, Mills JG, Johnson GE, Downs AR. Pop
entrapment syndrome. Ann Surg 1977;185:341-8.

4 Bouhotsos J, Daskalakis E. Muscular abnormalities affecting the popliteal vessel. Br f Surg 1981;68:501-6.

5 Cairols MA, Blanes I, Gimenez A, et al. An exceptional case of popliteal artery syndrome. Eur F Vasc Surg 1994;8:754-6. 6 Steurer J, et al. A new therapeutic approach to popliteal artery entrapment syndrome. Eur $\mathcal{F}$ Vasc Endovasc Surg 1995;10:243-7. 